\title{
ACTITUDES DE EMPRENDIMIENTO, NECESIDAD DE LOGRO Y LA INTENCIÓN DE DESARROLLAR UN NEGOCIO EN ESTUDIANTES DE UNIVERSIDADES PÚBLICAS DE LIMA METROPOLITANA
}

\author{
ENTREPRENEURSHIP ATTITUDES, NEED FOR ACHIEVEMENT, AND THE \\ INTENTION OF DEVELOPING A BUSINESS AMONG PUBLIC UNIVERSITY \\ STUDENTS IN LIMA METROPOLITANA
}

\author{
Alejandro Loli P¹., Javier Del Carpio G., Arturo Vergara V. \\ Universidad Nacional Mayor de San Marcos, Lima, Perú \\ (RECIBIDO 5/1/2012; ACEPTADo El 8/4/ 2012)
}

\begin{abstract}
RESUMEN
El objetivo del estudio es conocer las actitudes de emprendimiento, la necesidad de logro y la intención de desarrollar un negocio en estudiantes de las universidades públicas de Lima, en una muestra de 657 sujetos que estudian diversas especialidadeslos encuestados cursan entre el primer y décimo ciclo de estudios.

Los hallazgos encontrados nos indican que la mayoría de estudiantes tienen una actitud positiva de creatividad y emprendimiento; asimismo, hay una asociación altamente significativa de las actitudes de empredimiento con la intención de desarrollar un negocio y aquel con la necesidad de logro, disminuyendo dicha significancia al asociar la necesidad de logro con la intención de desarrollar un negocio. Igualmente, un análisis del puntaje total del emprendimiento con sus componentes arroja que existe una correlación significativa y positiva con todos ellos. Respecto de las variables demográficas, sociales y organizacionales y su relación con el emprendimiento (bajo, normal y alto), no se encuentran diferencias significativas cuando se comparan entre universidades, salvo cuando se trata de la experiencia laboral de los sujetos; tampoco existe asociación significativa con las variables como la edad, el género, el estado civil y ciclos de estudio.
\end{abstract}

Palabras clave: Actitudes, emprendimiento, intención de negocio, necesidad de logro, estudiantes universitarios.

\begin{abstract}
The objective of this study is to know the entrepreneurship attitudes, need for achievement, and the intention of developing a business among public university students in Lima Metropolitana. The study selected a sample of 657 students belonging to many majors, between the first semester and the tenth one.
\end{abstract}


The findings show us that the most of the students have a positive attitude to creativity and entrepreneurship. By the same token, there is a strong relationship between the entrepreneurshipattitudes to start a new business and the need for achievement. However, that relationship diminishes when it is analyzed the relationship between the need for achievement and the intention of developing a new business. In the same way, an analysis of entrepreneurship total score and its components shows that there is a positive and significant correlation among all of them. Regarding the demographic, social, and their relationship to the entrepreneurship (low, normal, high); it is not found that there are significant differences when it is compared the data form different universities, and the only exception appears when working experience of the subjects is analyzed on the other hnad, there is not a significant association among variables such as age, gender, marital status, and the study level.

Keywords: Attitudes, entrepreneurship, intention of a business, need for achievement, college students

\section{INTRODUCCIÓN}

La crisis económica y los conflictos político-sociales de las dos últimas décadas del siglo pasado trajeron como consecuencia la migración masiva de la población de las zonas rurales a las grandes ciudades, lo que generó desempleo y desplazamiento de las clases medias de la sociedad hacia niveles de pobreza nunca antes visto. La alternativa que optaron dichos migrantes y desempleados fue crear sus propias fuentes de trabajo, muchas de ellas, en el momento actual, lograron pasar de una situación informal a otra de naturaleza formal y operan con éxito como pequeñas, medianas empresas familiares.

A este fenómeno se denomina "emprendimiento", donde la capacidad creativa se puso en juego y activó otras competencias como la iniciativa, la perseverancia, la necesidad de logro, etc. permitiéndoles encontrar solución a sus problemas. Carpio (1999) decía que la creatividad describe una "tendencia a comportarse creativamente"... y que se puede identificar a través de la observación de "colecciones de eventos conductuales"..., y que se logra con el entrenamiento en la solución de problemas, convirtiéndose estas en promotoras del comportamiento creativo. Sin duda que las experiencias sucesivas de carencias y soluciones de los migrantes y desocupados no era sino una forma de entrenamiento y de aprendizaje por error.

El emprendedor es aquel que detecta una oportunidad, la analiza, la estudia y se proyecta para crear una organización, es aquella persona que posee olfato y que sabe encontrar las oportunidades, es alguien que cree en sus ideas y que es capaz de llevarlas a cabo, de encontrar la manera de materializar sus sueños; alguien que aprende de sus errores, que no se inmoviliza o abandona cuando se equivoca o fracasa, que intenta salir adelante pese a las dificultades que encuentra. Obviamente, aprender a emprender no fue nada fácil, pero las circunstancias donde las alternativas son escasas, sin duda, juegan un papel motivador importante para no amilanarse (M. Rodríguez, 2007). 
Se dice que el empredendor “... es un innovador, un gran estratega, (...) tiene personalidad creativa, siempre desafiando lo desconocido, transformando posibilidades en oportunidades” (Gerber, 1996, en J. Bóveda, 2004); “... emprededores pueden ser definidos como individuos que innovan, identifican y crean oportunidades de negocios ..." (Lezana y Tonelli, 1998, en J. Bóveda, 2004). De allí que se diga que los emprendedores "nacen y no se hacen", y es que las personas son también producto de los procesos de socialización, de manera que ambas corrientes de complementan.

La situación actual es de un nuevo escenario que sigue a la crisis, donde, de una u otra manera, todos los países han quedado involucrados y muchos de ellos están comprometidos en impulsar el emprendimiento, encontrando como el lugar más propicio los centros de estudios, especialmente universitarios, donde los jóvenes concentran las potencialidades creativas e innovadoras más que en ningún otro sector de la sociedad. Ya decía Senges, (2007) que "el emprendimiento no es algo de una persona o de un especialista: es una filosofía de vida que deberían adoptar las instituciones (...), lo que promueve la cultura emprendedora dentro la universidad es por un lado un modelo mental, y por otro una estructura práctica del día a día que da soporte a los que quieren emprender/innovar/optimizar". Por ello, se espera que estas iniciativas de emprendimiento perduren en el tiempo más que aquellas creadas sobre la base de las carencias y el desempleo.

Sin embargo, existen estudios a favor y en contra de los supuestos anteriores, tal es el caso de un estudio desarrollado por R. Espíritu y M. Sastre (2007) en una universidad de Madrid (no especificado en la fuente), con una muestra de 668 estudiantes, en la que se encontró que los estudiantes de más reciente incorporación a la universidad presentan tasas de actitud emprendedora superiores a las de sus compañeros más veteranos. Asimismo, aquellos que tienen una formación más específica tienden a ser más conservadores.

Gilberto Vejarano M. y Pilar Espinoza O. (2008) desarrollaron una investigación con el objeto de identificar y medir la actitud emprendedora de los estudiantes de la Universidad Tecnológica Equinoccial, Quito, Ecuador, a fin de diseñar estrategias de promoción, comunicación, capacitación e impulso al emprendimiento, en una muestra de 245 estudiantes. Encontraron que existen actitudes y conductas altamente positivas hacia el emprendimiento, así como de apoyo a las estrategias que conlleven a fortalecer las actividades que conduzcan a fomentar una cultura emprendedora.

El estudio de N. Espinoza (2004), sobre aptitudes y actitudes empresariales de los estudiantes de administración en tres universidades públicas de Lima y Callao, en una muestra de 200 alumnos, encontró que el 56.5\% no tienen trabajo dependiente, y el $43.5 \%$ tiene trabajo dependiente. En el primer caso, el $16.81 \%$, indica tener negocio propio y $83.19 \%$, no tener negocio propio; en el segundo caso, el $9.5 \%$ señala tener negocio propio, y el resto no tiene negocio propio.

Aquellos que no trabajan ni tienen negocio propio (47\%) tienen como objetivo concluir sus estudios $(27.65 \%)$, realizar sus prácticas profesionales $(26.59 \%)$, 
crear su negocio propio (25.55\%), buscar trabajo (17.02\%) y salir al extranjero (3.19\%). Datos con los que el autor trata de demostrar la escasa actitud de emprendimiento de los estudiantes, explicando que aquellos que dicen tener negocio propio se refieren al negocio de sus padres. No existen estrategias pedagógicas en las facultades de administración de las universidades públicas, no están orientadas a formar empresarios ni a promover aptitudes y actitudes empresariales.

Daniel Mavila Hinojoza y col. (2009) en un estudio que tiene por objeto conocer los factores influyentes en la capacidad emprendedora de los alumnos de la Universidad Nacional Mayor de San Marcos, en una muestra de 449 estudiantes del primer ciclo de estudios, descubrieron que existen cuatro factores que explican la capacidad de emprendimiento de los jóvenes ingresantes a la universidad: capacidad de relacionarse socialmente, creatividad, capacidad de planificación y capacidad de realización personal.

Alejandro E. Loli Pineda y cols. (2008) dan cuenta de una investigación desarrollada sobre la actitud emprendedora de los estudiantes de la Universidad Nacional Mayor de San Marcos (Perú) y su relación con algunas variables demográficas en una muestra de 182 sujetos pertenecientes a 20 facultades. En ella hallaron que la mayoría de estudiantes tiene una actitud positiva hacia la creatividad y el emprendimiento, y que existe una relación significativa con variables de edad, ciclo de estudios, Facultad al que pertenecen, desarrollo de emprendimiento y sector de actividad para emprender.

Una réplica del estudio anterior (A.Loli y col. 2009), en una muestra de 202 estudiantes de la Universidad Nacional de Ingeniería (Perú), permitió confirmar que la actitud hacia la creatividad y el empredimiento es positiva. Así mismo, la correlación entre la creatividad y los componentes del emprendimiento arroja una asociación significativa y positiva. Sobre las variables demográficas y sociales y su relación con los niveles del emprendimiento (bajo, normal y alto), existe diferencia significativa de acuerdo a los ciclos de estudio en los que se encuentran, la experiencia laboral, la intención de desarrollar un negocio y la necesidad de asistencia financiera; es decir, la intervención de dichas variables permiten inferir diferencias de percepción en los niveles de creatividad y el empredimiento.

Un diagnóstico del potencial emprendedor realizado en la Universidad Católica del Perú, en una muestra de 460 estudiantes del $4^{\circ}$ y $10^{\circ}$ ciclo de estudios, informa que el $71 \%$ de los estudiantes planeaba formar una empresa y que el $6 \%$ ya contaba con una empresa, aunque no indica si estas eran propias o de la familia (Dino Linares, 2001).

Otro estudio desarrollado con estudiantes universitarios es de F. A. Delicio (2007), su objeto es conocer acerca de la existencia de una vocación emprendedora entre los estudiantes y las motivaciones que llevan a dicha vocación en la Facultad de Ciencias Económicas y Sociales, de la Universidad Nacional de Mar del Plata UNMDP, Argentina. Se contó con una muestra 47 sujetos del último año de la carrera de contador público y administración. Los resultados nos indican que la proporción de estudiantes con vocación emprendedora es baja (14.89\%); el ejemplo 
familiar como modelo a seguir para desarrollar una vocación emprendedora entre los estudiantes es escasa; la experiencia laboral y la formación académica que reciben de la universidad es un buen complemento para intentar su propio emprendimiento, pero insuficiente; los alumnos demandan más cuestiones relacionadas con la posibilidad de desarrollar una actividad emprendedora. La universidad está preparando alumnos para la gran empresa, que no es precisamente la principal generadora de empleo.

Fernando Graña y Mariel Fornoni (2002) realizaron una investigación sobre la vocación emprendedora en una muestra de 189 sujetos de las Facultades de Ciencias Económicas y Sociales de dos universidades del Mar del Plata, Argentina, y encontraron que el $35,5 \%$ de los estudiantes posee vocación emprendedora; el $17,5 \%$ tienen comportamiento emprendedor, iniciando una empresa propia; el $8 \%$ tienen intención emprendedora, con un proyecto concreto para crear una nueva empresa; y el $10 \%$ tienen una actitud emprendedora, admitiendo la posibilidad de iniciar un nuevo negocio como opción de carrera o salida laboral.

Hugo Kantis y colaboradores (2000) dan cuenta de un estudio realizado para explorar las diferencias existentes en el proceso emprendedor de los egresados universitarios y aquellos que no ingresan a la universidad. Trabajaron en una muestra 122 fundadores de nuevas empresas en Argentina, encontrando que las empresas fundadas por emprendedores de base universitaria se distinguen por su perfil más calificado en materia de dinamismo, recursos humanos y del tipo de oportunidades que aprovechan. Así mismo, fue posible identificar algunas lecciones de interés por el apoyo de universidades al proceso emprendedor, también se descubrió que la participación de las mujeres, de aquellos que viven en áreas locales y de quienes provienen de familias de sectores medios bajos y bajos es limitado.

El Centro de Desarrollo Emprendedor del Instituto de Estudios Empresariales de Montevideo (IEEM) de la Universidad de Montevideo (2007), en el marco del proyecto Global Enterpreneurship Monitor (GEM), publicó algunos resultados sobre una investigación realizada sobre las relaciones entre la actividad emprendedora y el crecimiento económico en estudiantes universitarios. Según el informe, 13 de cada 100 personas estaban desarrollando, a mediados de 2006, algún tipo de empresa naciente. Según el PBI per cápita, estas cifras colocan a Uruguay en el séptimo lugar dentro del ranking de actividad emprendedora para los "países pobres". Sin embargo, cuando se le pregunta a los jóvenes iqué ha hecho usted en los últimos 12 meses para poder emprender su negocio?, la mayoría dijo nada. Situación muy común en jóvenes universitarios, cuya reacción es básicamente actitudinal, de optimismo, y donde la Universidad debe jugar un papel más activo para el fomento de la actitud emprendedora (M. Olivero, 2007).

Juan A. Moriano L. (2005), por otro lado, desarrolló una investigación con el objeto de explicar y predecir la intención de emprender, en una muestra de 2,000 estudiantes, la mayor parte de ellos residentes en Castilla y León, España. Los resultados nos muestran que solo el $13.5 \%$ de los participantes muestran mayor intención de desarrollar su carrera profesional a través del autoempleo que traba- 
jando por cuenta ajena; sin embargo, el estudio señala que solo el $4.11 \%$ crearán efectivamente su propia empresa. Se sigue pensando que la mejor opción laboral es conseguir un trabajo seguro para toda la vida.

Finalmente, de la información lograda por los autores en investigaciones desarrolladas en muestras parciales, previas al presente estudio, más la literatura revisada con el mismo objetivo, se puede inferir que los emprendedores surgen de diversa manera: como respuesta a las necesidades y carencias, como aprendizaje de sus interacciones sociales o académicas, y como condiciones innatas que son fortalecidas durante su desarrollo. Probablemente por eso se dice que todos los seres humanos somos emprendedores por naturaleza. Lo cierto es que hay pocas evidencias científicas en nuestro medio, pero suficientes como para creer que las universidades tienen que jugar un papel trascendente en la formación de jóvenes emprendedores; por ello, creímos interesante conocer las actitudes de emprendimiento, la necesidad de logro y la intención de desarrollar un negocio de estudiantes de las Universidades Públicas de Lima Metropolitana, Perú.

\section{MÉTODO}

\section{Muestra}

La población está constituida por estudiantes de cinco universidades públicas que se encuentran en la ciudad de Lima Metropolitana, de ellas se tomaron tres para formar parte de la muestra por ser las más representativas.

Finalmente, la muestra quedó conformada por 657 estudiantes de diversas carreras profesionales y pertenecientes a diferentes años de estudio. De la totalidad de la muestra el $32.72 \%$ fueron de sexo femenino y el $67.28 \%$ de sexo masculino; entre ellos, el $97.1 \%$ eran solteros, el $1.2 \%$ casados, el $0.3 \%$ divorciados, el $1.4 \%$ de unión libre. La distribución de la muestra de acuerdo a la edad fue la siguiente:

TABLA 1: Proporción de muestra de docentes por unidad académica

\begin{tabular}{lccccc}
\hline & \multicolumn{5}{c}{ Edad } \\
\cline { 2 - 5 } Universidades & $15-20$ & $21-25$ & $26-30$ & 31 a más & TOTAL \\
\hline U. Agraria & 85 & 161 & 13 & 3 & 262 \\
U. Ingeniería & 106 & 77 & 17 & 2 & 202 \\
U. San Marcos & 33 & 125 & 28 & 7 & 193 \\
\hline TOTAL & 224 & 363 & 58 & 12 & 657 \\
\hline
\end{tabular}

\section{Instrumento}

El instrumento fue construido por Alejandro E. Loli Pineda (2008), con el propósito de investigar la creatividad y el emprendimiento en muestras diversas. Dicho 
inventario está conformado por 12 factores: creatividad e iniciativa, optimismo, perseverancia, compromiso con sus convicciones, confianza en sí mismo, propensión al riesgo, capacidad de adaptación, liderazgo, independencia y autonomía, necesidad de logro, visión del futuro y competencias; haciendo un total de 94 ítems entre todos sus componentes, los cuales cuentan con seis alternativas de respuesta que van desde totalmente en desacuerdo (1) a totalmente de acuerdo (6).

En concordancia con el coeficiente Alpha de Cronbach, los diferentes factores o componentes cumplen con el criterio de estabilidad y confiabilidad, superando el mínimo (0.20) exigido para estos fines. La confiabilidad general lograda en el procesamiento de datos del presente instrumento en esta muestra fue de 0.95 ; por tanto, altamente estable y confiable. En el siguiente cuadro se puede observar el nivel de confiabilidad de cada componente.

TABLA 2: Niveles de confiabilidad de los componentes

\begin{tabular}{clc}
\hline $\mathbf{N}^{\mathbf{0}}$ & \multicolumn{1}{c}{ Factores o componentes } & Confiabilidad \\
\hline 1 & Creatividad e iniciativa & 0,70 \\
2 & Optimismo & 0,62 \\
3 & Perseverancia & 0,68 \\
4 & Compromiso & 0,38 \\
5 & Confianza & 0,72 \\
6 & Propensión al riesgo & 0,65 \\
7 & Capacidad de adaptación & 0,60 \\
8 & Liderazgo & 0,78 \\
9 & Independencia y autonomía & 0,56 \\
10 & Necesidad de logro & 0,58 \\
11 & Visión del futuro & 0,65 \\
12 & Competencias o habilidades & 0,74 \\
\hline \multicolumn{2}{c}{}
\end{tabular}

Cronbach's Alpha

\section{Procesamiento de datos}

Se utilizó el paquete estadístico SPSS (Statistical Package for the Social Sciences) para el tratamiento estadístico. El estadístico de confiabilidad Alpha de Cronbach se empleó para ver los niveles de confiabilidad de la prueba total y de cada uno de sus componentes, la correlación de Spearman para establecer el grado de significancia en la asociación entre el puntaje total de emprendimiento y sus componentes, y la prueba de chi-cuadrado para conocer las diferencias que pueden surgir al comparar los niveles de emprendimiento con la necesidad de logro y la intención de desarrollar un negocio y también con las variables psicosocio demográficas. 
Finalmente, con el objeto de conocer la actitud hacia el empredimiento, se utilizó el cuartil q1 y q3 como criterio de partición para determinar el grado de empredimiento (Bajo, Normal, Alto) a partir del puntaje obtenido por los participantes de la muestra.

\section{RESULTADOS}

\section{Las actitudes de emprendimiento de los estudiantes universitarios}

La Tabla 3 nos indica las actitudes de creatividad y emprendimiento de los estudiantes de las universidades públicas de Lima Metropolitana, donde se puede apreciar, tomando en cuenta los doce componentes del inventario, que existe una actitud positiva, en la medida en que la mayoría de estudiantes de la muestra se encuentra dentro de la categoría normal (49.9\%) y alto $(25.1 \%)$, correspondiendo a la categoría baja el $25.0 \%$. Si bien este último porcentaje no supera a la categoría alta, debe ser motivo de preocupación de las autoridades universitarias para superar estos niveles de percepción de los estudiantes.

TABLA 3: Actitudes de emprendimiento de estudiantes universitarios

\begin{tabular}{cccc}
\hline $\begin{array}{c}\text { Niveles de emprendi- } \\
\text { miento }\end{array}$ & Frecuencia & $\begin{array}{c}\text { Porcentaje } \\
\text { válido }\end{array}$ \\
\hline \multirow{4}{*}{ Válidos } & Bajo & 164 & 25.0 \\
& Normal & 328 & 49.9 \\
& Alto & 165 & 25.1 \\
\hline & Total & 657 & 100.0 \\
\hline
\end{tabular}

\section{Asociación entre el emprendimiento y sus componentes}

En la siguiente tabla, encontramos el análisis de correlación del puntaje total de las actitudes hacia la creatividad y el emprendimiento con las actitudes hacia cada uno de sus componentes de los estudiantes de las universidades públicas de Lima; en él se observa que existe una correlación significativa y positiva entre el emprendimiento y la creatividad e iniciativa $\left(\mathrm{r}=.84^{* * *}\right)$, el optimismo $\left(\mathrm{r}=.78^{* * *}\right)$, la perseverancia $\left(\mathrm{r}=.78^{* * *}\right)$, el compromiso con sus convicciones $(\mathrm{r}=.64 * * *)$, la confianza en sí mismo $\left(\mathrm{r}=.83^{* * *}\right)$, la propensión al riesgo $\left(\mathrm{r}=.75^{* * *}\right)$, la capacidad de adaptación $\left(\mathrm{r}=.77^{* * *}\right)$, el liderazgo $\left(\mathrm{r}=.78^{* * *}\right)$, la independencia y autonomía $\left(\mathrm{r}=.61^{* * *}\right)$, la necesidad de logro $\left(\mathrm{r}=.71^{* * *}\right)$, la visión del futuro $(\mathrm{r}=.79 * * *)$ y las competencias o habilidades $(\mathrm{r}=.79 * * *)$; es decir, cuanto mayor es la creatividad e iniciativa, optimismo, perseverancia, compromiso con sus convicciones, confianza en sí mismo, propensión al riesgo, capacidad de adaptación, liderazgo, independencia y autonomía, necesidad de logro, visión del futuro y competencias o habilidades, mayor es la actitud de emprendimiento de los estudiantes universitarios. 
Como se puede apreciar, esta asociación confirma la actitud positiva de los estudiantes de las universidades públicas de Lima frente a la creatividad y el emprendimiento, probablemente se debe a las expectativas que genera la formación profesional de la mayoría de ellos y la posibilidad de ejercerla de manera independiente.

TABLA 4: Correlación entre el puntaje total de emprendimiento con sus componentes

\section{Factores de emprendimiento}

\begin{tabular}{|c|c|c|}
\hline \multirow[t]{2}{*}{ Creatividad e iniciativa } & Coeficiente de correlación & 0.84 \\
\hline & Sig. (bilateral) & 0.00 \\
\hline \multirow[t]{2}{*}{ Optimismo } & Coeficiente de correlación & 0.78 \\
\hline & Sig. (bilateral) & 0.00 \\
\hline \multirow[t]{2}{*}{ Perseverancia } & Coeficiente de correlación & 0.78 \\
\hline & Sig. (bilateral) & 0.00 \\
\hline \multirow[t]{2}{*}{ Compromiso con sus convicciones } & Coeficiente de correlación & 0.64 \\
\hline & Sig. (bilateral) & 0.00 \\
\hline \multirow[t]{2}{*}{ Confianza en sí mismo } & Coeficiente de correlación & 0.83 \\
\hline & Sig. (bilateral) & 0.00 \\
\hline \multirow[t]{2}{*}{ Propensión a los riesgos } & Coeficiente de correlación & 0.75 \\
\hline & Sig. (bilateral) & 0.00 \\
\hline \multirow[t]{2}{*}{ Capacidad de adaptación } & Coeficiente de correlación & 0.77 \\
\hline & Sig. (bilateral) & 0.00 \\
\hline \multirow[t]{2}{*}{ Liderazgo } & Coeficiente de correlación & 0.78 \\
\hline & Sig. (bilateral) & 0.00 \\
\hline \multirow[t]{2}{*}{ Independencia y autonomía } & Coeficiente de correlación & 0.61 \\
\hline & Sig. (bilateral) & 0.00 \\
\hline \multirow[t]{2}{*}{ Necesidad de logro } & Coeficiente de correlación & 0.71 \\
\hline & Sig. (bilateral) & 0.00 \\
\hline \multirow[t]{2}{*}{ Visión del futuro } & Coeficiente de correlación & 0.79 \\
\hline & Sig. (bilateral) & 0.00 \\
\hline \multirow[t]{2}{*}{ Competencias o habilidades } & Coeficiente de correlación & 0.79 \\
\hline & Sig. (bilateral) & 0.00 \\
\hline
\end{tabular}

Puntaje total de emprendimiento 


\section{Las actitudes de emprendimiento y la intención de desarrollar un negocio de los estudiantes universitarios}

En la Tabla 5, encontramos la relación entre los niveles de emprendimiento y la intención de desarrollar un negocio ( $\mathrm{p}$-value $=0.00$ ), situación que conduce a inferir que existen diferencias significativas en la percepción de los estudiantes sobre la intención de desarrollar un negocio respecto del nivel de emprendimiento. Es decir, en todos los niveles de emprendimiento (bajo, normal y alto) hay la intención de desarrollar un negocio, más acentuado en los niveles normal y alto; contrariamente, si bien en todos los niveles de emprendimiento, también hay quienes no tienen intención de desarrollar un negocio, estos son mayores en aquellos de nivel de emprendimiento bajo.

Los estudiantes que presentan un nivel de emprendimiento normal, el $96 \%$ tiene la intención de desarrollar un negocio; aquellos que tienen un nivel de emprendimiento alto, el $98.8 \%$ tiene la intención de desarrollar un negocio; incluso los que presentan un nivel de emprendimiento bajo, el $87.2 \%$ tienen la intención de desarrollar un negocio. Esta relación probablemente se debe a que las personas que tienen una actitud positiva hacia la creatividad y el emprendimiento, siempre o casi siempre estarán pendientes de emprender un negocio, incluso aquellos estudiantes que tienen un nivel de emprendimiento bajo tienen interés en desarrollar un negocio, lo que podría estar indicando su convicción por iniciar un emprendimiento si se presentara la oportunidad.

TABLA 5: Niveles de emprendimiento y la intención de desarrollar un negocio

\begin{tabular}{lllll}
\hline \multirow{2}{*}{$\begin{array}{l}\text { Niveles de emprendi- } \\
\text { miento }\end{array}$} & & \multicolumn{3}{c}{$\begin{array}{c}\text { Intención de desarrollar un } \\
\text { negocio }\end{array}$} \\
\cline { 3 - 4 } & & & si & \multicolumn{2}{c}{ notal } \\
\hline Bajo & Sujetos & 143 & 21 & 164 \\
& $\%$ & $87.2 \%$ & $12.8 \%$ & $100.0 \%$ \\
\multirow{2}{*}{ Normal } & Sujetos & 315 & 13 & 328 \\
& $\%$ & $96.0 \%$ & $4.0 \%$ & $100.0 \%$ \\
Alto & Sujetos & 163 & 2 & 165 \\
& $\%$ & $98.8 \%$ & $1.2 \%$ & $100.0 \%$ \\
\hline Total & Sujetos & 621 & 36 & 657 \\
& $\%$ & $94.5 \%$ & $5.5 \%$ & $100.0 \%$ \\
\hline
\end{tabular}

p-value $<0.01$ (Chi-Square likehood)

\section{El emprendimiento y la necesidad de logro de los estudiantes universitarios}

En la Tabla 6, se observa la relación entre los niveles de emprendimiento y los niveles de necesidad de logro $(\mathrm{p}$-value $=0.00)$, hecho que estaría indicando que existen diferencias significativas en la percepción de los estudiantes de acuerdo a los niveles de necesidad de logro respecto de las actitudes de emprendimiento. Es decir, en todos los niveles de emprendimiento (bajo, normal y alto) existe la 
necesidad de logro, siendo alto en los niveles de emprendimiento bajo y normal, excepto cuando el nivel de emprendimiento es alto, la necesidad de logro es igualmente alta.

Los estudiantes que presentan un nivel de emprendimiento bajo tienen una necesidad de logro normal de $89.6 \%$ y alta de $4.3 \%$, los de nivel de emprendimiento normal tienen una necesidad de logro normal de $69.5 \%$ y alta de $28.7 \%$, y aquellos de nivel de emprendimiento alto tienen una necesidad de logro normal de $23.6 \%$ y alta $76.4 \%$. Esta diferencia podría indicar que, aunque el nivel de emprendimiento sea bajo habrá siempre una expectativa por alcanzar logros profesionales, claro está que aquellos que tienen niveles de emprendimiento normal y alto tienen mayor disposición de alcanzar logros en el futuro.

TABLA 6: Niveles de emprendimiento según niveles de necesidad de logro

\begin{tabular}{|c|c|c|c|c|c|}
\hline \multirow{2}{*}{$\begin{array}{l}\text { Niveles de em- } \\
\text { prendimiento }\end{array}$} & & \multicolumn{3}{|c|}{ Necesidad de logro } & \multirow{2}{*}{ Total } \\
\hline & & Bajo & Normal & Alto & \\
\hline \multirow[t]{2}{*}{ Bajo } & Sujetos & 10 & 147 & 7 & 164 \\
\hline & $\%$ & $6.1 \%$ & $89.6 \%$ & $4.3 \%$ & $100.0 \%$ \\
\hline \multirow[t]{2}{*}{ Normal } & Sujetos & 6 & 228 & 94 & 328 \\
\hline & $\%$ & $1.8 \%$ & $69.5 \%$ & $28.7 \%$ & $100.0 \%$ \\
\hline \multirow[t]{2}{*}{ Alto } & Sujetos & 0 & 39 & 126 & 165 \\
\hline & $\%$ & $.0 \%$ & $23.6 \%$ & $76.4 \%$ & $100.0 \%$ \\
\hline \multirow[t]{2}{*}{ Total } & Sujetos & 16 & 414 & 227 & 657 \\
\hline & $\%$ & $2.4 \%$ & $63.0 \%$ & $34.6 \%$ & $100.0 \%$ \\
\hline
\end{tabular}

p-value $<0.01$ (Chi-Square likehood)

\section{La necesidad de logro y la intención de desarrollar un negocio de los estu- diantes universitarios}

En la Tabla 7, se puede apreciar la relación entre los niveles de necesidad de logro y la intención de desarrollar un negocio ( $\mathrm{p}$-value $=0.048$ ), situación que indica que existen diferencias en la percepción de los estudiantes entre los niveles de necesidad de logro y la intención de desarrollar un negocio. Es decir, en todos los niveles de necesidad de logro (bajo, normal y alto) existe intención de desarrollar un negocio, siendo más alto en el nivel de necesidad de logro alto, en cambio cuando el nivel de necesidad de logro es normal y bajo, la intención de desarrollar un negocio es ligeramente baja.

Los estudiantes que presentan un nivel de necesidad de logro bajo tienen la intención de desarrollar un negocio en un $93.8 \%$ y no tienen la intención de desarrollar un negocio en un $6.3 \%$, los de nivel de necesidad de logro normal tienen la intención de desarrollar un negocio en un $93.0 \%$ y no tienen la intención de desarrollar un negocio en un $7.0 \%$, y aquellos de nivel de necesidad de logro alto tienen 
la intención de desarrollar un negocio en un $97.4 \%$ y no tienen la intención de desarrollar un negocio en un $2.6 \%$.

Las diferencias antes señaladas, a pesar de ser ligeramente significativas, podrían indicar que en todos los niveles de necesidad de logro, la mayoría de los estudiantes tienen la disposición de emprender un negocio, pero también existen de aquellos que tienen necesidad de logro pero no tienen intención de desarrollar un negocio. Estos últimos, probablemente estén pensando en incursionar, entre otras, en empleos dependientes y burocráticos sean estos públicos o privados.

TABLA 7: Niveles de la necesidad de logro y la intención de desarrollar un negocio

\begin{tabular}{clccc}
\hline \multirow{2}{*}{ Necesidad de logro } & & \multicolumn{2}{c}{$\begin{array}{c}\text { Intención de desarrollar un } \\
\text { negocio }\end{array}$} & \multirow{2}{*}{ Total } \\
\cline { 2 - 4 } & & sí & no & \\
\cline { 2 - 4 } Bajo & Sujetos & 15 & 1 & 16 \\
& $\%$ & $93.8 \%$ & $6.3 \%$ & $100.0 \%$ \\
\multirow{2}{*}{ Normal } & Sujetos & 385 & 29 & 414 \\
& $\%$ & $93.0 \%$ & $7.0 \%$ & $100.0 \%$ \\
\multirow{2}{*}{ Alto } & Sujetos & 221 & 6 & 227 \\
& $\%$ & $97.4 \%$ & $2.6 \%$ & $100.0 \%$ \\
\hline Total & Sujetos & 621 & 36 & 657 \\
& $\%$ & $94.5 \%$ & $5.5 \%$ & $100.0 \%$ \\
\hline
\end{tabular}

p-value $<0.05$ (Chi-Square likehood)

\section{Actitudes de emprendimiento y las variables demográficas, sociales y orga- nizacionales}

Respecto de las variables demográficas, sociales y organizacionales y su relación con los niveles de emprendimiento y otras variables intervinientes en la muestra estudiada, se encontró que hay asociación cuando se analiza los grupos de estudiantes por universidades y también según la experiencia laboral lograda por ellos, como se podrá apreciar en los siguientes cuadros; sin embargo, no encontramos el mismo comportamiento respecto de la edad, el género, el estado civil, el sector donde piensa desarrollar su emprendimiento, las áreas de capacitación para el emprendimiento y la necesidad financiera, probablemente se debe a que estas variables son independientes a la creatividad y el emprendimiento o la intención de desarrollar un negocio y su presencia no influye, no cambia la percepción de los estudiantes. Vale decir, que probablemente los factores de influencia estén ligados más a razones personales, familiares, no confían que la universidad pueda hacer algo al respecto o ven aún distante para tomar esas decisiones.

\section{Actitudes de emprendimiento de los estudiantes por universidades}

En la Tabla 8, se muestra la relación de los niveles de emprendimiento según universidades $(p$-value $=0.132$ ), lo que indica que no existen diferencias en la 
percepción de los estudiantes entre los niveles de emprendimiento según universidades a las que pertenecen. Es decir, los estudiantes de las tres universidades no se diferencian o son homogéneas respecto de los niveles de emprendimiento (bajo, normal y alto). Lo que permite inferir que esta variable no influye en la percepción de los estudiantes respecto del emprendimiento.

La mayoría de los estudiantes de la Universidad Nacional Agraria La Molina tiene un nivel de emprendimiento normal de $53.8 \%$, alto de $23.3 \%$ y bajo de $22.9 \%$; la mayoría de los estudiantes de la Universidad Nacional de Ingeniería tiene un nivel de emprendimiento normal de $50.0 \%$, alto de $21.8 \%$ y bajo de $28.2 \%$; mientras que la mayoría de los estudiantes de la Universidad Nacional Mayor de San Marcos tiene un nivel de emprendimiento normal de $44.6 \%$, alto de $31.1 \%$ y bajo $24.4 \%$. Si comparamos las tres universidades podría decirse que los estudiantes de la Universidad de Ingeniería tienen el nivel de emprendimiento más bajo y los de la Universidad de San Marcos el más alto.

Esta homogeneidad podría indicar que en las tres universidades prevalecen las mismas virtudes y defectos y demandan de cambios para una percepción diferenciada de los estudiantes sobre sus futuras acciones profesionales; es decir, desde cambios curriculares orientados a promover la creatividad y el emprendimiento, pasando por la calidad académica, hasta el soporte administrativo y de gestión que faciliten altos niveles de rendimiento.

TABLA 8: Niveles de emprendimiento por universidades

\begin{tabular}{|c|c|c|c|c|c|}
\hline \multirow{2}{*}{ Universidades } & & \multicolumn{3}{|c|}{ Niveles de emprendimiento } & \multirow{2}{*}{ Total } \\
\hline & & Bajo & Normal & Alto & \\
\hline \multirow{2}{*}{ U. Agraria } & Sujetos & 60 & 141 & 61 & 262 \\
\hline & $\%$ & $22.9 \%$ & $53.8 \%$ & $23.3 \%$ & $100.0 \%$ \\
\hline \multirow{2}{*}{ U. Ingeniería } & Sujetos & 57 & 101 & 44 & 202 \\
\hline & $\%$ & $28.2 \%$ & $50.0 \%$ & $21.8 \%$ & $100.0 \%$ \\
\hline \multirow{2}{*}{ U. San Marcos } & Sujetos & 47 & 86 & 60 & 193 \\
\hline & $\%$ & $24.4 \%$ & $44.6 \%$ & $31.1 \%$ & $100.0 \%$ \\
\hline \multirow{2}{*}{ Total } & Sujetos & 164 & 328 & 165 & 657 \\
\hline & $\%$ & $25.0 \%$ & $49.9 \%$ & $25.1 \%$ & $100.0 \%$ \\
\hline
\end{tabular}

p-value $>0.1$ (Chi-Square likehood)

\section{Actitudes de emprendimiento de los estudiantes y la experiencia laboral}

La Tabla 9 indica la relación entre la experiencia laboral lograda por los estudiantes y los niveles de emprendimiento ( $p$-value $=0.00$ ), por lo que se puede inferir que existen diferencias significativas en la percepción de los estudiantes de acuerdo a la experiencia laboral respecto del emprendimiento. 
Los estudiantes que declaran haber tenido experiencia laboral, el $29.7 \%$ se ubica en el nivel de emprendimiento alto, el $49.8 \%$ en el nivel normal y el $20.5 \%$ en el nivel bajo; mientras que aquellos que declaran no haber tenido experiencia laboral, el $16.1 \%$ se ubica en el nivel de emprendimiento alto, el $50.2 \%$ en el nivel normal y el $33.6 \%$ en el nivel bajo.

Lo que indica que la experiencia laboral está asociada a mejores niveles de emprendimiento, contrariamente a aquellos que dicen no haber tenido experiencia laboral; sin embargo, el porcentaje de estudiantes que se ubican en niveles de emprendimiento normal y alto, a pesar de no tener experiencia, puede estar indicando la expectativa que tienen para emprender un negocio. De allí, la actitud positiva hacia el emprendimiento.

TABLA 9: Niveles de emprendimiento según la experiencia laboral

\begin{tabular}{clcccr}
\hline \multirow{2}{*}{ Experiencia laboral } & & \multicolumn{3}{c}{ Niveles de emprendimiento } & \multirow{2}{*}{ Total } \\
\cline { 3 - 5 } & & Bajo & Normal & Alto & \\
\hline \multirow{2}{*}{ No ha tenido experiencia } & Sujetos & 75 & 112 & 36 & 223 \\
& $\%$ & $33.6 \%$ & $50.2 \%$ & $16.1 \%$ & $100.0 \%$ \\
\multirow{2}{*}{ Si ha tenido experiencia } & Sujetos & 89 & 216 & 129 & 434 \\
& $\%$ & $20.5 \%$ & $49.8 \%$ & $29.7 \%$ & $100.0 \%$ \\
\hline \multirow{2}{*}{ Total } & Sujetos & 164 & 328 & 165 & 657 \\
& $\%$ & $25.0 \%$ & $49.9 \%$ & $25.1 \%$ & $100.0 \%$ \\
\hline
\end{tabular}

p-value $<0.01$ (Chi-Square likehood)

\section{La necesidad de logro de los estudiantes por universidades}

En la Tabla 10 se muestra la relación de la necesidad de logro por universidades $(p$-value $=0.276)$, por lo que se puede inferir que no existen diferencias en la percepción de los estudiantes de acuerdo a la universidad a la que pertenecen respecto de la necesidad de logro. En otros términos, la pertenencia a una universidad u otra no influye en la percepción de los estudiantes sobre la necesidad de logro.

Los estudiantes que indican pertenecer a la Universidad Nacional Agraria de la Molina, el 63.0\% se ubica en el nivel normal de necesidad de logro, el $34.7 \%$ en el nivel alto y el $2.3 \%$ en el nivel bajo; mientras que aquellos que declaran pertenecer a la Universidad Nacional de Ingeniería, el $64.9 \%$ se ubica en el nivel normal, el $31.2 \%$ en el nivel alto y el $4.0 \%$ en el nivel bajo. Así mismo, los estudiantes que dicen pertenecer a la Universidad Nacional Mayor de San Marcos, el 61.1\% se encuentran en el nivel normal de necesidad de logro, el 37.8\% se ubican en el nivel alto y el $1.0 \%$ en el nivel bajo.

Este hallazgo, a pesar de no ser significativa, estaría indicando que la necesidad de logro es más baja en los estudiantes de la Universidad de Ingeniería, mejorando entre los estudiantes de la Universidad Agraria y siendo más alta entre los estudiantes de la Universidad Nacional Mayor de San Marcos. 
TABLA 10: Relación de los niveles de necesidad de logro por universidades

\begin{tabular}{clcccr}
\hline \multirow{2}{*}{ Universidades } & \multicolumn{3}{c}{ Niveles de necesidad logro } & \multirow{2}{*}{ Total } \\
\hline \multirow{2}{*}{ U. Agraria } & Sujetos & 6 & Normal & Alto & \\
& $\%$ & $2.3 \%$ & $63.0 \%$ & $34.7 \%$ & $100.0 \%$ \\
U. Ingeniería & Sujetos & 8 & 131 & 63 & 202 \\
& $\%$ & $4.0 \%$ & $64.9 \%$ & $31.2 \%$ & $100.0 \%$ \\
U. San Marcos & Sujetos & 2 & 118 & 73 & 193 \\
& $\%$ & $1.0 \%$ & $61.1 \%$ & $37.8 \%$ & $100.0 \%$ \\
\hline \multirow{2}{*}{ Total } & Sujetos & 16 & 414 & 227 & 657 \\
& $\%$ & $2.4 \%$ & $63.0 \%$ & $34.6 \%$ & $100.0 \%$ \\
\hline
\end{tabular}

p-value $>0.10$ no es significativo

\section{La intención de desarrollar un negocio según universidades}

En la Tabla 11 se encuentra la relación entre la intención de desarrollar un negocio de los estudiantes por universidades $(\mathrm{p}$-value $=0.853$ ), lo que lleva a inferir que no existe diferencia significativa en la percepción de los estudiantes cuando se analiza por grupos de procedencia respecto de la intención de desarrollar un negocio. Es decir, pertenecer a una universidad o a otra no tiene mucho que ver con la intención de desarrollar un negocio, es independiente.

Los estudiantes que indican que tienen intención de desarrollar un negocio, el 94.7\% pertenecen a la Universidad Nacional Agraria de La Molina, 95.0\% pertenecen a la Universidad de Ingeniería, y el $93.8 \%$ pertenecen a la Universidad Nacional Mayor de San Marcos, mientras que aquellos que no piensan desarrollar un negocio, el 5.3\% son de la Universidad Agraria, 5.0\% son de la Universidad de Ingeniería, y el $6.2 \%$ son de la Universidad de San Marcos. Esta homogeneidad de los datos permite inferir que no existe influencia en la decisión de los estudiantes para emprender un negocio. Sin embargo, se puede observar que la intención de desarrollar un negocio es ligeramente superior a las otras universidades.

TABLA 11: Relación de las universidades y la intención de desarrollar un negocio de los estudiantes

\begin{tabular}{cl|c|c|c}
\hline \multirow{2}{*}{ Universidad } & \multicolumn{2}{|c|}{ Intención de desarrollar un negocio } & \multirow{2}{*}{ Total } \\
\cline { 3 - 4 } & & si & no & \\
\hline U. Agraria & Sujetos & 248 & 14 & 262 \\
& $\%$ & $94.7 \%$ & $5.3 \%$ & $100.0 \%$ \\
U. Ingeniería & Sujetos & 192 & 10 & 202 \\
& $\%$ & $95.0 \%$ & $5.0 \%$ & $100.0 \%$ \\
San Marcos & Sujetos & 181 & 12 & 193 \\
& $\%$ & $93.8 \%$ & $6.2 \%$ & $100.0 \%$ \\
Total & Sujetos & 621 & 36 & 657 \\
& $\%$ & $94.5 \%$ & $5.5 \%$ & $100.0 \%$ \\
\hline
\end{tabular}

p-value $>0.10$ no es significativo 


\section{La experiencia laboral y la intención de desarrollar un negocio de los estu- diantes}

En la Tabla 12 se encuentra la relación entre la experiencia laboral y la intención de desarrollar un negocio de los estudiantes ( $\mathrm{p}$-value $=0.003$ ), lo que lleva a inferir que existe diferencia significativa en la percepción de los estudiantes de acuerdo a la experiencia respecto de la intención de desarrollar un negocio.

Los estudiantes que indican no tener experiencia, el 30.7\% dicen que sí tiene la intención de desarrollar un negocio y el 3.2\% dice no tener esa intención; mientras que aquellos que indican haber tenido experiencia el $63.8 \%$ tiene la intención de desarrollar un negocio y el $2.3 \%$ no tiene tal intención.

Lo que estaría indicando que la experiencia juega un papel importante en la decisión de los estudiantes, y que aún los que no tienen experiencia tienen la expectativa de emprender.

TABLA 12: Relación entre la experiencia laboral y la intención de desarrollar un negocio de los estudiantes

\begin{tabular}{clccr}
\hline \multirow{2}{*}{ Experiencia laboral } & & \multicolumn{2}{c}{$\begin{array}{c}\text { Intención de desarrollar } \\
\text { un negocio }\end{array}$} & \multirow{2}{*}{ Total } \\
\cline { 3 - 4 } & & sí & no & \\
\hline \multirow{2}{*}{ no ha tenido experiencia } & Sujetos & 202 & 21 & 223 \\
& $\%$ & $30.7 \%$ & $3.2 \%$ & $33.9 \%$ \\
\multirow{2}{*}{ si ha tenido experiencia } & Sujetos & 419 & 15 & 434 \\
& $\%$ & $63.8 \%$ & $2.3 \%$ & $66.1 \%$ \\
\hline Total & Sujetos & 621 & 36 & 657 \\
& $\%$ & $94.5 \%$ & $5.5 \%$ & $100.0 \%$ \\
\hline
\end{tabular}

p-value $<0.01$ (Fisher para una tabla de $2 \times 2$.)

\section{DISCUSIÓN}

Bajo el supuesto que los jóvenes disponen de mejores potencialidades creativas y emprendedoras, se ha planteado la necesidad de conocer las actitudes de emprendimiento, la necesidad de logro y la intención de desarrollar un negocio de los estudiantes de las universidades públicas de Lima Metropolitana, en una coyuntura social, política y económica que atraviesa el Perú frente a la globalización de la economía y la competitividad de las organizaciones productivas y de servicio, y la necesidad de incrementar emprendimientos sostenibles.

Desde luego, esta investigación como la revisión de las investigaciones ha estado orientada hacia el sector de estudiantes de nivel universitario que aspiran una carrera profesional a corto plazo.

De los resultados obtenidos se puede deducir que la mayoría de los estudiantes de las universidades públicas en Lima - Perú tienen una actitud positiva hacia el emprendimiento, ubicándose en el nivel normal y alto, al igual que los resultados encontrados por G. Vejarano y P. Espinoza (2008) en Quito - Ecuador. 
Entre tanto, hay una asociación significativa y positiva del emprendimiento con sus componentes, resultado que es coherente con lo encontrado por A. Loli y cols. (2008, 2009 y 2010), en estudios de muestras más reducidas, demostrando consistencia de dicha relación. En esa medida si consideramos que la actitud es una tendencia positiva o negativa -en este caso- hacia el emprendimiento, y siendo la creatividad un componente del emprendimiento hay una tendencia a comportarse creativamente (C. Carpio, 1999); así, los estudiantes universitarios con actitudes positivas tienen mayores posibilidades de iniciar un emprendimiento que aquel otro que no tiene esa actitud durante su formación profesional, donde generalmente ya existe entrenamiento teórico y práctico en la solución de problemas.

Ya decía M. Rodríguez (2007), “... el emprendedor es aquel que detecta una oportunidad, la analiza, la estudia y se proyecta para crear una organización", es aquella persona que posee olfato y que sabe encontrar las oportunidades, es alguien que cree en sus ideas y que es capaz de llevarlas a cabo, de encontrar la manera de materializar sus sueños; alguien que aprende de sus errores, que no se inmoviliza o abandona cuando se equivoca o fracasa, que intenta salir adelante pese a las dificultades que encuentra. Obviamente, aprender a emprender no es nada fácil, pero las circunstancias donde las alternativas son escasas y las posibilidades son mayores, sin duda, ellas juegan un papel motivador importante para no amilanarse. Siendo así, se espera que la actitud de los estudiantes de la muestra tenga su correlato en el comportamiento de ellos después de su formación profesional.

La creatividad describe una "tendencia a comportarse creativamente" y que se puede identificar a través de la observación de "colecciones de eventos conductuales", decía C. Carpio (1999), y que se logra con el entrenamiento en la solución de problemas, convirtiéndose estas en promotoras del comportamiento creativo, $\mathrm{y}$, por tanto, accesibles al aprendizaje y el emprendimiento. Desde este punto de vista, la asociación entre el emprendimiento y la intención de desarrollar un negocio sugiere que los centros superiores de formación profesional tienen un rol fundamental en el proceso de promoción del desarrollo de actitudes y comportamientos de emprendimiento desde la universidad, como afirma M. Olivero (2007) que la universidad debe jugar un papel más activo para el fomento de la actitud emprendedora. Senges, M. (2007), en cambio, va más allá y cree que "el emprendimiento no es algo de una persona o de un especialista: es una filosofía de vida que deberían adoptar las instituciones..."

Cuando se hace un análisis de los niveles de necesidad de logro en relación con la intención de desarrollar un negocio se encuentran diferencias en las actitudes y percepción de los estudiantes; es decir, existe solo infuencia relativa de los niveles de necesidad de logro respecto de la decisión de desarrollar un negocio. Probablemente debido a que los estudiantes piensan que el logro profesional no siempre está ligado al negocio sino que se puede conseguir en el ejecicio profesional dependiente, y porque “... la universidad está preparando alumnos para la gran empresa, que no es precisamente la principal generadora de empleo (F.A. Delicio, 2007). 
De todos modos, como lo demuestran otros estudios, las actitudes de los estudiantes universitarios no son sino tendencias, y pocos son los que tienen negocio propio y generalmente dichos negocios resultan siendo de los padres (Espinoza, 2004; Dino Linares, 2001; M. Olivero, 2007); la preocupación de ellos es fundamentalmente concluir sus estudios, hacer sus prácticas profesionales; excepto, en algunos casos como el que informa Graña y Fornoni (2002) y Juan A. y Moriano L. (2005), de comportamientos concretos de emprendimiento. Obviamente, esta condición se mantendrá mientras las universidades no promuevan cambios sustanciales en lo académico como en lo administrativo.

Respecto de la relación de las variables demográficas y organizacionales, en un análisis separado por universidades, se ha encontrado una débil asociación con los niveles de emprendimiento; es decir, estas diferencias, ligeramente significativas, de los niveles de emprendimiento por universidades, podrían indicar que en las tres universidades prevalecen las mismas virtudes y defectos y demandan de cambios en la percepción de los estudiantes sobre sus futuras acciones profesionales; es decir, desde cambios curriculares orientados a promover la creatividad y el emprendimiento, pasando por la calidad académica, hasta el soporte administrativo y de gestión que faciliten altos niveles de rendimiento.

Por otro lado, la relación entre los niveles de emprendimiento y la experiencia, indica que la experiencia influye en la percepción de los estudiantes respecto de los niveles de emprendimiento; de manera que, incluso existen estudiantes que a pesar de no tener experiencia, tienen expectativa para emprender un negocio. De allí que un análisis sobre la relación de la experiencia laboral y la intención de desarrollar un negocio de los estudiantes, igualmente significativa, confirman los hallazgos anteriores. Coherentemente con estos hallazgos F. A. Delicio (2007) encontró que la experiencia laboral y la formación académica que reciben de la universidad es un buen complemento para intentar su propio emprendimiento.

Finalmente, contrario a lo encontrado por A. Loli y cols (2008), en el presente estudio, no hay diferencias significativas de los niveles de emprendimiento, como tampoco de los niveles de necesidad de logro ni la intención de desarrollar un negocio cuando interviene la edad, el sexo, el estado civil y la carrera profesional; igualmente, cuando se pone en prueba un análisis por universidades no hay diferencias. Es decir, dichas variables no afectan la percepción de los estudiantes respecto del emprendimiento, la necesidad de logro y la intención de desarrollar un negocio.

Por consiguiente, de mejorar las condiciones ofrecidas por las universidades, sin duda, es probable que aquellos emprendedores que tienen una base universitaria se distingan por un perfil más calificado respecto del dinamismo, recursos humanos y aprovechamiento de oportunidades (Hugo Kantis, 2000); así, todos los esfuerzos deben orientarse a la promoción de actitudes y comportamientos creativos y emprendedores de los estudiantes. 


\section{CONCLUSIONES}

1. Tomando en cuenta la muestra total y los doce componentes del inventario, existe una actitud positiva, en la medida en que la mayoría de estudiantes se encuentra dentro de la categoría normal y alta correspondiendo a la categoría baja menos de la tercera parte de estudiantes.

2. Existe asociación entre las actitudes de los estudiantes hacia el emprendimiento con las actitudes de los mismos hacia cada uno de sus componentes o factores (creatividad e iniciativa, optimismo, perseverancia, compromiso con sus convicciones, confianza en sí mismo, propensión al riesgo, capacidad de adaptación, liderazgo, independencia y autonomía, necesidad de logro, visión del futuro y competencias o habilidades).

3. Existen diferencias significativas en la percepción de los estudiantes sobre la intención de desarrollar un negocio respecto del nivel de emprendimiento. Probablemente se debe a que las personas que tienen una actitud positiva hacia el emprendimiento, siempre o casi siempre están pendientes de emprender un negocio, incluso aquellos estudiantes que tienen un nivel de emprendimiento bajo tienen interés en desarrollar un negocio, lo que podría estar indicando su convicción por iniciar un negocio si se presentara la oportunidad.

4. Existen diferencias significativas en la percepción de los estudiantes de acuerdo a los niveles de necesidad de logro respecto de las actitudes de emprendimiento. Esta diferencia podría indicar que, aunque el nivel de emprendimiento sea bajo habrá siempre una expectativa por alcanzar logros profesionales, claro está que aquellos que tienen niveles de emprendimiento normal y alto tienen mayor disposición de alcanzar logros en el futuro.

5. Existen diferencias en la percepción de los estudiantes entre los niveles de necesidad de logro y la intención de desarrollar un negocio. Si bien es cierto que, en todos los niveles de necesidad de logro, la mayoría de los estudiantes tienen la disposición de emprender un negocio, también hay de aquellos que tienen necesidad de logro pero no tienen intención de desarrollar un negocio. Estos últimos probablemente estén pensando en incursionar, entre otras, en empleos dependientes y burocráticos sean públicos o privados.

6. Existen diferencias significativas en la percepción de los estudiantes de acuerdo a la experiencia laboral respecto del emprendimiento. Probablemente debido a que la experiencia laboral está asociada a mejores niveles de emprendimiento, contrariamente a aquellos que dicen no haber tenido experiencia laboral; sin embargo, el porcentaje de estudiantes que se ubican en niveles de emprendimiento normal y alto, a pesar de no tener experiencia, puede estar indicando la expectativa que tienen para emprender un negocio.

7. Existen diferencias significativas en la percepción de los estudiantes de acuerdo a la experiencia respecto de la intención de desarrollar un negocio. Lo que 
estaría indicando que la experiencia juega un papel importante en la percepción de los estudiantes; de allí que aún los que no tienen experiencia tienen la expectativa de emprender, llegado el momento.

8. La relación de las variables tales como la edad, el sexo, el estado civil, facultad de pertenencia, universidad de procedencia, entre otros, con los niveles de emprendimiento, los niveles de necesidad de logro y la intención de desarrollar un negocio no son significativas, llevándonos a la conclusión, que la participación de dichas variables no afectan o no cambian en nada la percepción de los estudiantes. Vale decir, que probablemente los factores de influencia estén ligados más a razones personales, familiares, o ven aún distante para tomar esas decisiones.

\section{RECOMENDACIONES}

De los resultados obtenidos y el análisis de los mismos se puede inferir que es posible elaborar y desplegar en todas las universidades del país, programas de intervención a fin de promover e impulsar mejoras en la sociedad, tales como:

1. Mantener y mejorar las actitudes de emprendimiento de los estudiantes de las universidades, implulsando reformas curriculares orientadas a la creatividad y el emprendimiento en todas las carreras profesionales.

2. Impulsar el despliegue de la mejora continua de la calidad académica con visión de futuro en las universidades.

3. Crear e implementar incubadoras de empresas dentro de las universidades a fin de fortalecer y apoyar actitudes emprendedoras y concretar la intención de desarrollar un negocio.

4. Generar condiciones para la implementación de Parques Tecnológicos, si es necesario en alianza con otras universidades, gobiernos locales y/o regionales.

5. Impulsar la implantación de programas de formación de emprededores en los niveles básicos de educación (inicial, primaria y secundaria), a través de los Centros de Extensión y Proyección Social de las universidades en coordinación con el Ministerio de Educación.

\section{REFERENCIAS BIBLIOGRÁFICAS}

Bóveda, Juan (2004). El emprendedor y las pequeñas empresas. Fundación EMPREDER. Rev. Futuros, Vol II(6). Paraguay: http://www.revistafuturos.inf Carpio, Claudio (2008). Competencias profesionales del Psicólogo. UNAM, México.

Centro de Desarrollo Emprendedor del Instituto de Estudios Empresariales de Montevideo (IEEM) (2007). Emprendedorismo en los universitarios: Una opción cada día más real. Montevideo: Universidad de Montevideo. 
Cevallos V. Emilio (2007). Restricciones del entorno a la competitividad empresarial en América Latina. Cochabamba, Bolivia: FUNDES Internacional.

Delicio, F. (2007). La vocación emprendedora de los estudiantes universitarios: El Caso de los estudiantes de la Facultad de Ciencias Económicas y Sociales de la Universidad Nacional de Mar del Plata. UNMDP. Mar del Plata, Argentina.

Espinoza, N. (2004). Las aptitudes y actitudes empresariales de los estudiantes de administración en las universidades públicas de Lima y Callao. Lima: Facultad de Ciencias Administrativas, UNMSM.

Espíritu, Roberto y Sastre, Miguel Castillo (2007). La actitud emprendedora durante la vida académica de los estudiantes universitarios. Cuadernos de Estudios Empresariales, vol. 17 95-116. Universidad Complutense de Madrid, España.

Freire, Andy (2004). Pasión por emprender: De la idea a la cruda realidad. Editorial Aguilar. Buenos Aires.

Graña, F. y Fornoni, M. (2002). La vocación emprendedora en los alumnos universitarios avanzados de Ciencias Económicas de la ciudad de Mar del Plata. Mar del Plata, Argentina: UNMDP.

http://www.ute.edu.ec/noticias/catedra/pages/art0016.html

Loli Pineda, A.; Del Carpio, J. y La Jara, E. (2008). "El emprendimiento en los estudiantes de la UNMSM y su relación con algunas variables sociodemográficas". Revista de Investigaciones Psicológicas, Vol. 12, $\mathrm{N}^{\mathrm{o}}$ 1, UNMSM, Lima, Perú.

Loli Pineda, A.; Dextre, J. E.; Del Carpio, J. y La Jara, E. (2009). "Actitudes de creatividad y emprendimiento en estudiantes de la Universidad Nacional de Ingenieria y su relación con algunas variables sociodemográficas". Rev. IIPSI, Vol. 14, Nº 2, UNMSM, Lima Perú.

Mavila, Daniel; Tinoco, Óscar y Campos César (2009). "Factores influyentes en la capacidad emprendedora de los alumnos de la Universidad Nacional Mayor de San Marcos". Rev. Producción y Gestión, Vol. 12(2), pp. 32-39 (2009), Facultad de Ingeniería Industrial, UNMSM, Lima, Perú.

Moriano. J. (2005). "El perfil psicosocial del emprendedor". Rev. Ministerio de Trabajo y Asuntos Sociales. Madrid: CES Colección Estudios.

Shiersmann, S.; Graña, F. y Liseras, N. (2002). Vocación emprendedora en alumnos universitarios avanzados: El caso de las Facultades de Ciencias Económicas de FASTA y de la UNMDP. VII Reunión anual red Pymes Mercosur, Rafaela. Publicación en cd-rom.

Silva, Jorge E. (2008), Emprendedor: Crear su propia empresa. Editorial Alfaomega, México.

Vejarano, Gilberto y Espinosa, Pilar. (2008). "Actitudes de los estudiantes de la Universidad Tecnológica Equinoccial hacia el emprendimiento". Cátedra, Rev. Digital Universitaria $\mathrm{N}^{\circ} 2$, Año 1, Publicación semestral. UTE, Quito. 
